\title{
Expression of a Kallikrein-like Protease from the Snake Venom: Engineering of Autocatalytic Site in the Fusion Protein to Facilitate Protein Refolding
}

\author{
Chin-Chun Hung* and Shyh-Horng Chiou*', ${ }^{\prime 1}$ \\ *Institute of Biochemical Sciences, College of Science, National Tai wan University, Taiwan; and \\ †I nstitute of Biological Chemistry, P.O. Box 23-106, Academia, Taipe, Taiwan
}

Received J uly 19, 2000

In order to circumvent the difficulty encountered in the expression and purification of the recombinant products in E. coli system, we have developed a novel and facile method of removing the polyhistidine tag from target proteins after heterologous gene expression. The expression of a serine protease (Tm-5) from Taiwan habu (Trimeresurus mucrosquamatus) is taken as an exemplar to illustrate the basic rationales and protocols involved. In place of an enterokinase recognition site, a polyhistidine tag linked to an autocatalyzed site based on cleavage specificity of the serine protease flanking on the $5^{\prime}$-end of $\mathrm{Tm}-5$ clone sequence was engineered before protein expression in E. coli system. Renaturation of the fusion protein after expression revealed that the recombinant protease had refolded successfully from the inclusion bodies. Upon autocleavage of the expressed protease, the polyhistidine tag with additional amino acid residues appended to the $\mathbf{N}$-terminus of the coding sequence is found to be removed accordingly. The protein expressed and purified by this new strategy possesses a molecular weight of approximately 28,000 in accord with the expected value for this venom protease. F urther characterization of the recombinant protein employing a variety of techniques which include immunoblot analysis, RP-HPLC, ESI-MS, and N-terminal amino acid sequencing all shows indistinguishable properties to those of the isolated native protease. Most noteworthy is that the recombinant $\mathrm{Tm}-5$ protease also exhi bits amidase activity against $\mathrm{N}$-benzoylPro-Phe-Arg-p-nitroanilide, a unique and strict substrate for native Tm proteases reported previously. (c) 2000 Academic Press

The cDNA sequence for one of the precursors (Tm-5) of venom serine proteases from Taiwan habu (Trimeresurus mucrosquamatus) has been deposited in the EMBO database under Accession No. X83221.

${ }^{1}$ To whom correspondence should be addressed. Fax: (886)-226530014. E-mail: shchiou@gate.sinica.edu.tw.
Key Words: heterologous gene expression; fusion protein; polyhistidine tag; enterokinase recognition site; protein refolding; venom protease.

Expression and purification of recombinant proteins from host organisms are often a critical and timeconsuming task in achieving the goal of obtaining pure and large quantities of proteins from recombinant sources (1). A facile removal of contaminant expression proteins is essential to the accurate characterization of functional properties of a cloned protein. The most common solution to this problem is to engineer the expressed protein product so as to contain additional amino acids which convey a unique property to the protein of interest that can be exploited for purification purposes. Such a strategy would greatly increase the availability of recombinant proteins for further structural and functional study. The property generally chosen is to impart to a protein with a few additional amino acids for their binding ability to transition metal ions, thereby allowing proteins to be purified using immobilized metal ion affinity chromatography (IMAC) (2). In this method a specific chelating peptide can be cloned onto the amino terminus of a recombinant protein to serve as a purification tag or handle (3) and subsequently purified by using I MAC.

Recent advance in recombinant DNA technology has allowed in vitro fusions of genes or gene fragments in a simple and predictable manner. There are several reasons to use gene fusions for expression of recombinant proteins in heterologous hosts. In particular, a more reliable and reproducible method to obtain a native protein might be to use in vitro cleavage of the fusion protein, as compared to in vivo removal of the formylmethionine or cleavage of a signal peptide, which in both cases may yield a heterogeneous $\mathrm{N}$ terminus. It is noteworthy that many expression vectors currently used encode a protease cleavage site that allows re 
lease of carboxyl-terminal fusion partners from fusion proteins without leaving unwanted amino acid residues on their amino termini. In previous reports of snake venom protein expression, removal of the upstream fusion partners had been done with one of three different proteases, including thrombin $(4,5)$, factor Xa $(6,7)$, and enterokinase (8). However in our expression of a serine protease (Tm-5) from Taiwan habu (Trimeresurus mucrosquamatus) (9), we have repeatedly encountered some difficulty to remove the attached $\mathrm{N}$-terminal polyhistidine tag due to the inability of the commercial enterokinase to cleave efficiently the desired product from the expressed fusion venom protein. In this study, we have therefore designed and constructed expressed fusion venom protein. In this study, we have therefore designed and constructed some novel plasmid vectors for venom protease expression. We show that this novel method can obtain highly purified and active protein by refolding the recombinant protein (Tm-5) from inclusion bodies expressed in the $E$. coli system. The autolytic cleavage of venom protease in situ from the polyhistidine-tagged fusion protein by the fused protease itself results in an active recombinant form of $\mathrm{Tm}-5$ with identical properties to the authentic natural protease.

\section{MATERIALS AND METHODS}

Materials. The synthetic substrates were from Sigma Chemical Co. (St. Louis, MO). Restriction endonucleases, pfu polymerase and isopropyl- $\beta$-D-thiogalactoside (IPTG) were obtained from Promega (Promega Corp., Madison, WI). Antibodies directed against kallikrein-like fibrinogenolytic enzymes were purified from a rabbit antiserum, obtained by immunization with Tm-VIG from Taiwan habu (9).

Construction of recombinant Tm-5 expression plasmids. Polymerase chain reaction was employed to construct a recombinant protease corresponding to Tm-5 clone. Oligonucleotide A, a 39-mer (5'GCGGATCCGATGACGATGACAAAGTCATTGGAGGTGATG) for Tm-5 clone containing the enterokinase recognition site (boldface) and oligonucleotide B, a 33-mer (5'-GCGGATCCXXXXXXXXXXGTCATTGGAGGTGATG) for Tm-5 clone containing the autolytic recognition site (boldface $X X-$ ) were synthesized. They both include the $\mathrm{N}$-terminal coding sequence (underlined), and BamHI site (italicized).

Oligonucleotide C, a 26-mer (5'-GCCTGCAGTCACAGGGGGCAGGTTAC) was complementarily overlapped with the C-terminal end of Tm-5, which contained the Pstl site (italicized) linked to the translational stop codon (boldface) at the C-terminal end of the coding region (underlined).

Expression and purification of recombinant Tm-5 protease A nucleotide segment coding for one of the Tm proteases ( $\mathrm{Tm}-5)$ was synthesized by amplifying Tm-fibrinogenase open reading frame of the cDNA clone Tm5 using two primers designed for introducing cleavage sites for BamHI and Pstl plus a $6 \times \mathrm{His}$ tag and an $\mathrm{N}$-terminal extension of pentapeptide (Asp) ${ }_{4}$-Lys, or an N-terminal extension of tripeptides designed for autolytic cleavage by the expressed protease. This PCR-synthesized nucleotide segment was cloned in-frame into the BamHI/Pstl sites of the E. coli expression vector pQE 30 (Qiagen GmbH, Hilden, Germany). The deduced expressed $\mathrm{N}$-terminal sequences are shown in Fig. 1. Recombinant plasmids were identified by BamHI/Pstl analysis and clones contain- ing the correctly sized insert were sequenced. The $25 \mu \mathrm{g} / \mathrm{ml}$ kanamycin and $100 \mu \mathrm{g} / \mathrm{ml}$ ampicillin. For induction of gene expression, $\mathrm{E}$. coli M15 cells containing recombinant plasmids were grown at $37^{\circ} \mathrm{C}$ in one liter of LB medium containing $25 \mu \mathrm{g} / \mathrm{ml}$ kanamycin and 100 $\mu \mathrm{g} / \mathrm{ml}$ ampicillin. When the $A_{600 \mathrm{~nm}}$ of the growing culture reached 0.7 , IPTG was added to a final concentration of $2 \mathrm{mM}$. The culture was induced for a period of up to $4 \mathrm{~h}$.

Purification of histidinetagged recombinant Tm-5 protease Culture containing IPTG-induced cells $(1000 \mathrm{ml})$ was centrifuged and the pellet was resuspended ( $5 \mathrm{ml}$ per gram) in denaturation buffer ( 8 $\mathrm{M}$ urea, $0.5 \mathrm{M} \mathrm{NaCl}, 0.02 \mathrm{M} \mathrm{Tris} / \mathrm{HCl}, \mathrm{pH}$ 8.0). The lysed cells were stirred for $1 \mathrm{~h}$ at room temperature. The suspension was then centrifuged at $10,000 \mathrm{~g}$ for $15 \mathrm{~min}$ at $4^{\circ} \mathrm{C}$. The supernatant of centrifuged lysate was then loaded onto a $4 \mathrm{ml}$ nickel nitrilotriacetic acid ( $\mathrm{Ni}$ NTA) resin packed column (Qiagen $\mathrm{GmbH}$ ) preequilibrated with denaturation buffer and washed with washing buffer $(40 \mathrm{mM}$ imidazole in denaturation buffer) followed by elution buffer (1 M imidazole in denaturation buffer). The desired fusion proteins were analyzed by SDS-PAGE and Western blot analysis.

Immunoblot analysis of the expressed recombinant protease SDS-polyacrylamide slab gel (5\% stacking/15\% resolving gel) electrophoresis (SDS-PAGE)) was routinely carried out as described (10). For immunoblot screening of expressed Tm-5 protease, the gel was subjected to el ectrobl otting onto a nitrocellul ose membrane after SDS-PAGE followed by using a polyclonal rabbit anti-TmVIG antibody (9) as primary antibody and reacted with peroxidaseconjugated goat anti-rabbit IgG + IgM (H + L) (J ackson ImmunoResearch Laboratories, West Grove, PA). A color development reaction was carried out using diaminobenzidine and hydrogen peroxide.

Characterization of recombinant Tm-5 protease. The His-tagged Tm-fusion protein eluted from Ni-NTA resin was diluted with $50 \mathrm{mM}$ Tris, pH 8.0, buffer containing $5 \mathrm{mM}$ cysteine- $\mathrm{HCl}$ to a final protein concentration of $0.01-0.05 \mathrm{mg} / \mathrm{ml}$. The refol ding process was allowed to proceed at room temperature and was monitored by assaying the amidolytic activity of recombinant Tm-5 on chromogenic substrate $\mathrm{N}$-benzoyl-Pro-Phe-Arg-pNA. The refolded fusion protein was concentrated in an Amicon cell concentrator with constant stirring under nitrogen pressure and purified further on a Sephadex-G 75 (Pharmacia, Uppsala, Sweden) column, peaks with activity were collected and purified by FPLC (fast performance liquid chromatography) in a Mono Q (HR 5/5) column with Amersham/Pharmacia Biotech equipment (Pharmacia, Uppsala, Sweden). Eluted proteins purified from the last step were treated (i) with enterokinase in 50 $\mathrm{mM}$ Tris, $\mathrm{pH} 7.5$ to remove $6 \times \mathrm{H}$ is tag and $\mathrm{N}$-terminal extension of pentapeptide (Asp) ${ }_{4}$-Lys, or (ii) without enterokinase, i.e., autolytic cleavage of histidine tag and the attached $\mathrm{N}$-terminal extension tripeptides by the expressed protease. The concentration of Tm-5 venom protease was determined by the Bio-Rad protein assay kit.

Chromogenic assays. The enzyme activity of expressed venom proteases was determined by using chromogenic substrates. The amidolytic activity towards the chromogenic substrates was measured with a Ultrospec 4000 spectrophotometer (Amersham/ Pharmacia) in a plastic cuvette with 1-cm path length. Assays were performed in $50 \mathrm{mM}$ Tris- $\mathrm{HCl}, \mathrm{pH} 8.0$, in a total volume of $750 \mu \mathrm{l}$ at $37^{\circ} \mathrm{C}$. The final concentration of enzymes varied from 1.0 to $2.0 \mathrm{nM}$ and that of the chromogenic substrates from 0.1 to $0.3 \mathrm{mM}$. The formation of p-nitroaniline was monitored at $405 \mathrm{~nm}$ as a function of time.

$\mathrm{N}$-terminal amino acid sequence analyses. The unfolded or refolded recombinant $\mathrm{Tm}-5$ protease purified from His-tagged affinity resin was applied to a reversed-phase HPLC $\left(V_{\text {ydac }} \mathrm{C}_{4}\right.$ column, $4.6 \times$ $250 \mathrm{~mm})$. The HPLC was run in a linear gradient of $10-75 \%$ solvent B (95\% acetonitrile containing $0.1 \%$ trifluoroacetic acid (TFA)) with $5 \%$ acetonitrile/0.1\% TFA (solvent $A$ ) as the starting and equilibration eluent. The flow rate of column eluates was set at $1 \mathrm{ml} / \mathrm{min}$ and monitored at UV $280 \mathrm{~nm}$. Peak fractions were collected and lyophi- 
1 ATG AGA GGA TCG CAT CAC CAT CAC CAT CAC GGA TCC GAT GAC GAT GAC AAA GTC ATT GGA GGT GAT GAA Met Arg Gly Ser His His His His His His Gly Ser Asp Asp Asp Asp lys Val Ile Gly Gly Asp Glu

2 ATG AGA GGA TCG CAT CAC CAT CAC CAT CAC GGA TCC TTC CTG CGT GTC ATT GGA GGT GAT GAA Met Arg Gly Ser His His His His His His Gly Ser phe Leu Arg Val Ile Gly Gly Asp Glu

TTC GTC CGT

Phe Val Arg

4

TTC CCG CGT-

Phe Pro Arg

5

CCG TTC CGT

Pro Phe Arg

6

-CTG TTC CGT

Leu Phe Arg

FIG. 1. N-terminal extension sequences of various gene constructs and their corresponding amino acid sequences. They are composed of the $\mathrm{N}$-terminal coding sequence for the venom protease (underlined), the recognition site of enterokinase or the autolytic sites of Tm-VIG (boldface), and BamHI site (italicized) plus an initiating sequence (MRGS) from the expression vector. The dashed lines indicate identical sequence segments to those of sequences 1 and 2.

lized. N-terminal sequence analysis was carried out by automated Edman degradation with a microsequencing sequencer (M odel 477A, Applied Biosystems).

Molecular mass analyses. Expressed Tm-5 recombinant protease was dissolved in 50\% acetonitrile containing $1 \%$ acetic acid to make a final concentration of $0.1 \mu \mathrm{M}$. The sample was then analyzed in an LCQ mass spectrometer (Finnigan, San J ose, CA) at an infusion rate of $5 \mu \mathrm{l} / \mathrm{min}$. The spectra were analyzed with a software (LCQ BioWorks) from the manufacturer.

\section{RESULTS}

Gene fusion systems used to facilitate protein purification. We have adopted the common strategy of Smith et al. (3) by attaching an N-terminal polyhistidine tag to facilitate the affinity purification using immobilized metal ion affinity chromatography (IMAC) (2). However, in the final stage of removing the polyhistidine tag plus an N-terminal extension of pentapeptide (Asp) ${ }_{4}$-Lys by site-specific enterokinase, difficulties often arise due to the inability of obtaining clean cleavage of the attached affinity tag by the enzyme. In order to circumvent the difficulty encountered in the expression and purification of the recombinant products in E. coli system, we have therefore devel oped a novel strategy to facilitate the removal of attached tags.

For the expression and refolding of recombinant Tm-5 (rTm-5) in bacteria we used a novel strategy by making a synthetic fusion construct in E. coli expression vector $\mathrm{PQE} 30$, which contained the coding region for initiation translation peptide (Met-Arg-Gly-Ser), followed by a polyhistidine tag with a stretch of six histidines, a protease recognition site for an $\mathrm{N}$-terminal extension of pentapeptide (Asp) ${ }_{4}$-Lys or an N-terminal extension of tripeptides (Fig. 1) designed for

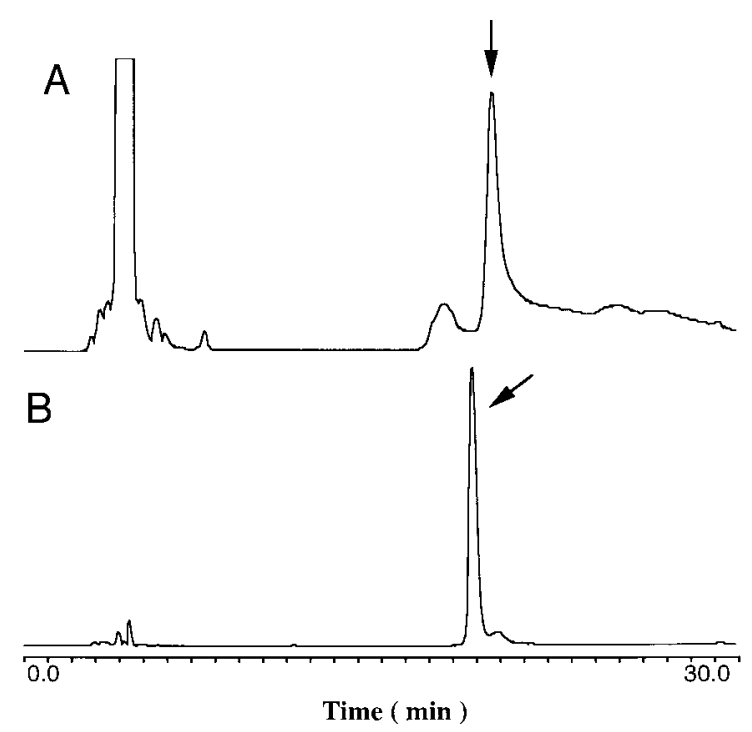

FIG. 2. Purification of recombinant $\mathrm{Tm}-5$ with histidine tag and tripeptide (Phe-Leu-Arg) extension by reversed-phase HPLC. The unfolded and refolded recombinant proteins isolated from $\mathrm{His}$-tagged affinity resin were applied to a reversed-phase $C_{4}$ column. The HPLC was run in a linear gradient of $10-75 \%$ solvent B (95\% acetonitrile containing $0.1 \%$ trifluoroacetic acid (TFA)) with $5 \%$ acetonitrile/ $0.1 \%$ TFA (solvent $A$ ) as the starting and equilibration eluent. The flow rate of column eluates was set at $1 \mathrm{ml} / \mathrm{min}$ and monitored at UV 280 $\mathrm{nm}$. Arrows indicate the unfolded rTm-5 (A) and refolded rTm-5 (B) proteases. 
A

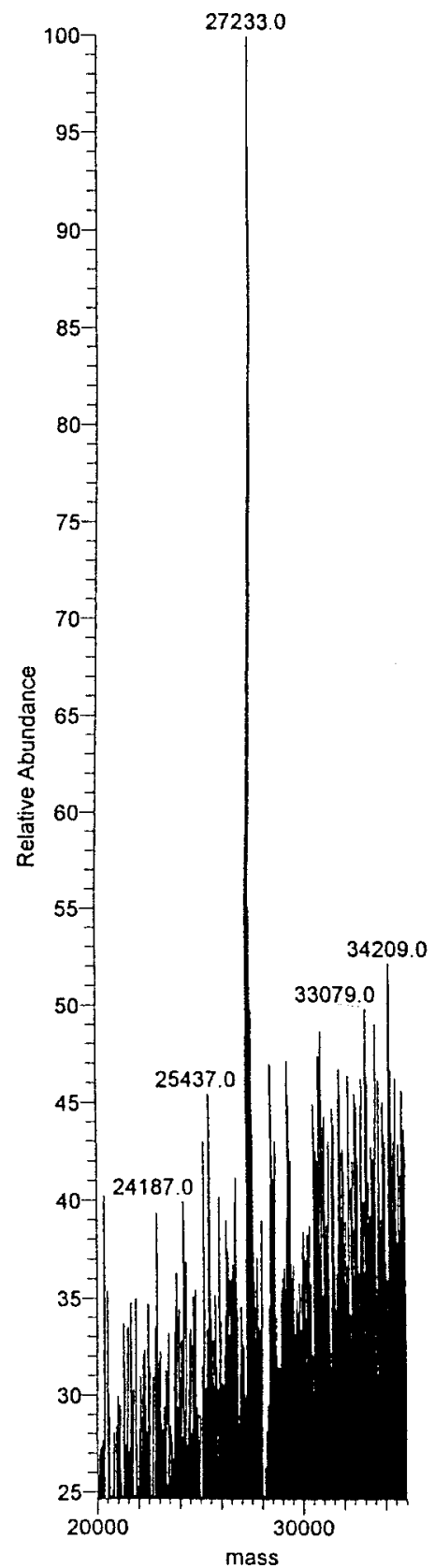

B

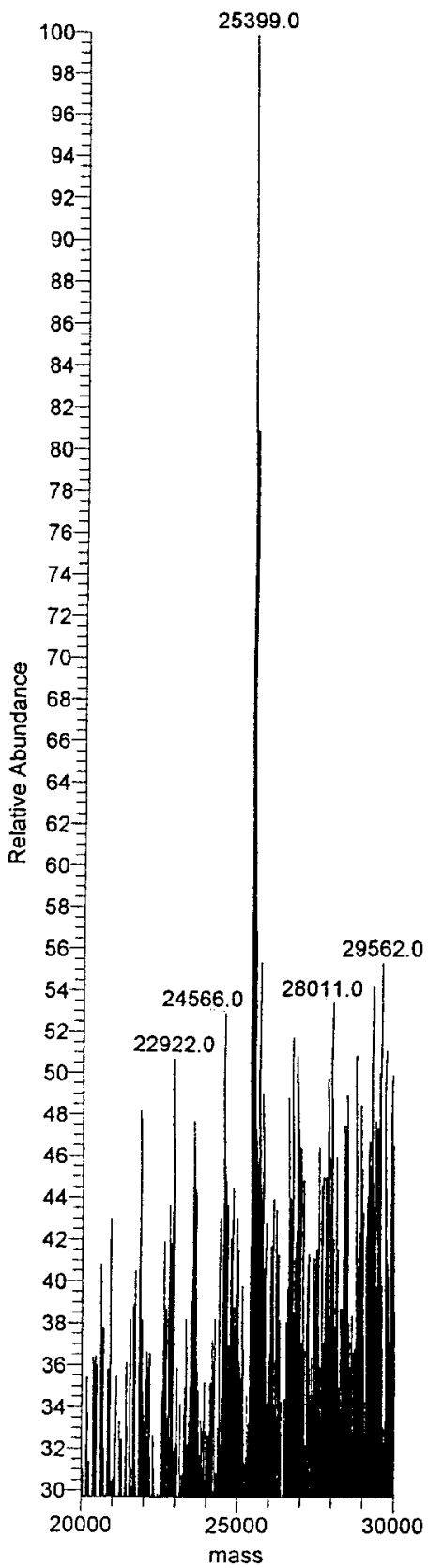

FIG. 3. The mass spectra of recombinant Tm-5 proteases as determined by el ectrospray ionization mass spectrometry. The molecular ions of unfolded (A) and refolded (B) from HPLC were detected with masses of 27233 and $25399 \mathrm{Da}$.

autolytic cleavage by the expressed protease, and ending with a sequence coding for rTm-5.

Expression and purification of recombinant $\mathrm{Tm}-5$ protease. The recombinant Tm-5 fusion protein was overexpressed in E. coli upon induction with IPTG. The expressed product was generated in a form of inclusion body with limited solubility. The polyhistidine tag facilitated purification of the rTm-5 fusion protein from the cell lysate in denaturation buffer containing $8 \mathrm{M}$ urea by immobilized nickel ion affinity chromatography (I MAC, Ni-NTA resin).

The refolding at the stage with fusion protein containing the polyhistidine tag followed by autolytic release of rTm-5 by the expressed protease was found to be more efficient than the original protocol of refolding the released product of $\mathrm{rTm}-5$ polypeptide from the fusion protein cleaved by enterokinase (11). In addition, we havealso found that enterokinase can degrade 


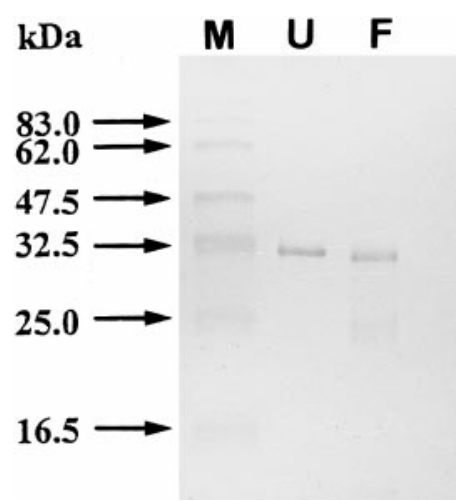

FIG. 4. I mmunoblot screening of expressed recombinant venom proteases. The gels were subjected to el ectroblotting onto a nitrocellulose membrane after SDS-PAGE followed by immunological analysis using rabbit polyclonal antibody against native Tm-VIG. Lane $M$, prestained standard protein markers, with 6 arrows on the left side showing the positions of markers; Iane $U$, unfolded recombinant Tm-5; lane F, refolded recombinant Tm-5.

unfolded polypeptide randomly before refolding (data not shown). The denatured and unfolded rTm-5 eluted from the Ni-NTA resin was then injected onto a $\mathrm{C}_{4}$ column. The elution profile (Fig. 2) showed that the unfolded rTm-5 was eluted later in the gradient than the refolded $r T m-5$. The eluted samples purified from HPLC were then analyzed by ESI-MS. The unfolded and refolded proteases were detected with masses of 27233 and $25399 \mathrm{Da}$, respectively (Fig. 3). The result clearly indicated that the fusion partner (an estimated mass of $1832 \mathrm{Da}$ ), including the His-tag appended to the $\mathrm{N}$-terminus of the $\mathrm{rTm}-5$ sequence, had been re moved successfully.

I mmunoblot analysis of recombinant Tm-5 protease. The predicted molecular mass of the mature TmVIG is about $25 \mathrm{kDa}$ based on the deduced protein sequences of cDNA clones, which is lower than $30 \mathrm{kDa}$ estimated by SDS-gel electrophoresis under reducing conditions. It is noteworthy that molecular-weight estimations by SDS-PAGE were slightly different between and extension peptides. However, both the unfolded and refolded rTm-5 proteases can cross-react with a polyclonal rabbit anti-TmVIG antibody by using immunoblot analysis (Fig. 4).

$\mathrm{N}$-terminal sequence analysis. N-terminal sequence analysis of various refolded $\mathrm{rTm}-5$ proteases with different $\mathrm{N}$-terminal extension peptides corroborated that these proteases had removed histidine-tagged fusion peptides by autolytic cleavage from recombinant rTm-5 fusion proteins (Table 1). All designed N-terminal extension peptides were cleaved to yield the correct $\mathrm{N}$-termini (VIGGDE) for this refolded recombinant protease except fusion proteins containing Pro-PheArg and Leu-Phe-Arg extension peptides, which produced one extra $\mathrm{N}$-terminus due to unexpected double cleavages by the expressed protease.
Substrate specificity of expressed recombinant Tm-5 proteases. In previous studies of activity assays for expressed proteases, we found that expressed products with intact histidine-tag and the attached pentapeptide possessed similar proteolytic activity to that of the native TmVIG enzyme using chromogenic substrates (data not shown). In this study, expressed recombinant proteases with removal of attached fusion peptides by autolysis were found to show similar activity to native venom proteases, both groups cleaving efficiently p-nitroanilide from several synthetic peptide substrates. As shown in Fig. 5, N-benzoyl-Pro-Phe-Arg p-nitroanilide, a specific substrate for kallikrein, was most susceptible to hydrolysis by rTm-5 (line 1 of Fig. 5). However, D-Val-Leu-Lys p-nitroanilide which is a substrate for plasmin was shown to be a poor substrate for rTm-5 (line 4 of Fig. 5). Enzyme activity assays of refolded recombinant protease $\mathrm{TTm}$-5 using $\mathrm{N}$-benzoylPhe-Val-Arg-pNA and N-p-tosyl-Gly-Pro-Arg-pNA (lines 2 and 3 of Fig. 5), both being substrates of thrombin, exhibit intermediate activity.

\section{DISCUSSION}

Snake venoms of the crotalid and viperid species are known to contain components with anticoagulant activity which strongly affect the haemostatic and blood coagulation system $(12,13)$. There have been diverse serine proteases isolated from various snake venoms. In our previous study, we have characterized a heatstable and active fibrinogenase from Taiwan habu (Trimeresurus mucrosquamatus), denoted as Tm-VIG, which possesses a strong and specific activity upon $\beta$-chains of fibrinogen (9). Comparison of substrate

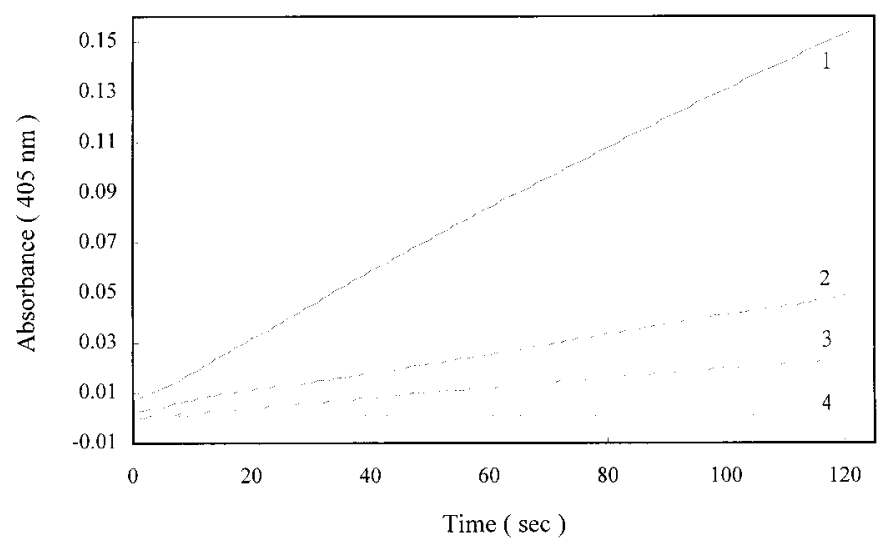

FIG. 5. Enzyme activity assays of recombinant Tm-5 on synthetic substrates. Activity was measured by using chromogenic substrates for rTm-5. Line 1, $0.1 \mathrm{mM}$ N-benzoyl-Pro-Phe-Arg-pNA and $0.2 \mu \mathrm{g}(0.1 \mathrm{nM})$ enzyme; line 2, $0.3 \mathrm{mM} N$-benzoyl-Phe-Val-Arg-pNA and $0.4 \mu \mathrm{g}$ enzyme $(0.2 \mathrm{nM})$; line 3, $0.3 \mathrm{mM} \mathrm{N}$-p-tosyl-Gly-Pro-Arg-pNA and $0.4 \mu \mathrm{g}$ enzyme $(0.2 \mathrm{nM})$; line 4, $0.3 \mathrm{mM} N$-p-tosyl-Gly-Pro-Lys-pNA and $0.4 \mu \mathrm{g}$ enzyme $(0.2 \mathrm{nM})$. Proteolytic activities using chromogenic substrates were measured on a spectrophotometer at $405 \mathrm{~nm}$. 


\section{TABLE 1}

Comparison of the Constructed N-Terminal Extension Peptide Sequences and N-Terminal Sequences Determined for Refolded Proteases

\begin{tabular}{cc}
\hline $\begin{array}{c}\text { Constructed Tm-5 } \\
\text { sequences }\end{array}$ & $\begin{array}{c}\text { N-terminal sequences } \\
\text { of refolded Tm-5 }\end{array}$ \\
\hline -FLRVIGGDE- & VIGGD \\
-FVRVIGGDE- & VIGGD \\
-FPRVIGGDE- & VIGGD \\
-PFRVIGGDE- & RVIGGD \\
-LFRVIGGDE- & VIGGD \\
& RVIGGD \\
& VIGGD \\
\hline
\end{tabular}

specificity between Tm-VIG and available venom serine proteases pointed to the fact that this venom protease with strong hypotensive effect on experimental animals may belong to the family of kallikrein-like proteases (unpublished results). Therefore generation of this venom enzyme in enough quantity for further mechanistic study and pharmacological assays may rest on the cloning and expression of this protease by application of DNA recombinant technology.

We have consistently encountered some difficulty when using the conventional protocol of removing the polyhistidine tag together with $\mathrm{N}$-terminal extension pentapeptide (Asp) ${ }_{4}$-Lys by enterokinase in the final stage of venom protease expression in $\mathrm{E}$. coli system. Therefore a novel strategy to facilitate the removal of attached tags and extension peptides has been developed in this report to effect the proficient expression and refolding of venom enzymes utilizing autolytic cleavage by the expressed protease itself instead of exogenous enterokinase.

The native Tm-VIG and the expressed venom fibrinogenase similar to reported kallikrein-like proteases from American crotalid snake (14), possess the highest catalytic efficiency towards the specific kallikrein substrate, N-benzoyl-Pro-Phe-Arg p-nitroanilide. However, unlike thrombin-like enzymes such as ancrod (15) and batroxobin (16), the expressed protease rTm-5 showed a low activity on hydrolyzing the ancrod substrate N-tosyl-Gly-Pro-Arg p-nitroanilide (Fig. 5). The enzyme did not hydrolyze X-X-Lys p-nitroanilide substrates, suggesting that arginine but not lysine is required at the $\mathrm{P} 1$ cleavage site for substrate recognition. Although $\mathrm{N}$-benzoyl-Pro-Phe-Arg p-nitroanilide is supposedly the best substrate for this venom protease, it is of interest to find that the expressed enzyme rTm-5 cleaves on both the $\mathrm{N}$ - and $\mathrm{C}$-sides of arginine leading to RVIGGD and VIGGD as two $\mathrm{N}$-termini of the expressed protein (Table 1). However this seems to be in agreement with our study of native peptide substrate specificity based on oxidized insulin B chain, which indicated that there are only three major susceptible sites to Tm-VIG, i.e. Leu ${ }^{15}-\mathrm{Tyr}^{16}{ }^{16} \mathrm{Arg}^{22}-\mathrm{Gly}^{23}$, and $\mathrm{Phe}^{25}-\mathrm{Tyr}^{26}$ (data not shown). Therefore we can obtain the clean expressed protein with VIGGDE as the only $\mathrm{N}$-terminus by employing tripeptides FLR, FVR or FPR as protease-cleavage sites. The designed tripeptide extension segments should have arginine situated at the $\mathrm{P} 1$ enzyme cleavage site and phenylalanine must be at the P3 instead of P2 site to effect the specific cleavage of $\mathrm{N}$-terminal extension peptide. This would provide some guidelines in the future application of this venom protease with certain advantage over enterokinase to protein expression and purification.

Expression of eucaryotic proteins in E. coli often resulted in the formation of insoluble inclusion bodies. Although we could obtain similar activities of recombinant fibrinogenases as compared with native Tm-VIG, the final yield of active recombinant fibrinogenases is about $2 \%$ or less. This may indicate a low efficiency in the refolding process. The presence of six pairs of disulfide poses a major problem for the expression of this venom protease during the refol ding process. Although the refolded venom enzyme possessed high proteolytic activities even if the six-histidine tag and $\mathrm{N}$-terminal extension pentapeptide (Asp) ${ }_{4}$-Lys were not removed. This finding is also consistent with other studies showing that six-histidine tag attached at $\mathrm{N}$ - or C-terminal of expressed proteins had little effect on the structure or function of final purified products $(17,18)$. Neverthel ess the proficient and complete removal of attached fusion peptides remains a major goal of protein expression and purification in view of great promise in the development of protein-based recombinant drugs. To our knowledge this is the first report on the design of nonconventional extension peptides coupled with autolysis of a specific venom protease to obtain a functional recombinant product free of histidinetag and fusion peptides.

\section{ACKNOWLEDGMENTS}

This work was supported in part by Academia and the National Science Council (NSC Grants 87-2311-B-002-068 and 88-2311-B-002061), Taipei, Taiwan. This report will be submitted as part of a dissertation by C.-C. Hung to National Taiwan University in partial fulfillment of the degree of Doctor of Philosophy. We thank Dr. Wei-Chao Chang at the Institute of Biological Chemistry, Academia, Taipei, Taiwan, for performing mass analysis of various recombinant proteases.

\section{REFERENCES}

1. Uhlén, M., and Moks, T. (1990) Methods Enzymol . 185, 129-143.

2. Porath, J ., Carlsson, J ., Olsson, I., and Belfrage, G. (1975) Nature 258, 598-599.

3. Smith, M. C., Furman, T. C., Ingolia, T. D., and Pidgeon, C. (1988) J . Biol. Chem. 263, 7211-7215.

4. Maeda, M., Satoh, S., Suzuki, S., Niwa, M., I oth, N., and Yamashina, I. (1991) J . Biochem. (Tokyo) 109, 632- 637.

5. Rosenthal, J. A., Levandoski, M. M., Chang, B., Potts, J . F., Shi, Q.-L., and Hawrot, E. (1999) Biochemistry 38, 7847-7855. 
6. Rosenthal, J . A., Hsu, S. H., Schneider, D., Gentile, L. N., Messier, N. J ., Vaslet, C. A., and Hawrot, E. (1994) J . Biol. Chem. 269, 11178-11185.

7. Zhang, Y., Wisner, A., Maroun, R. C., Choumet, V., Xiong, Y., and Bon, C. (1997) J . Biol. Chem. 272, 20531-20537.

8. Moura-da-Silva, A. M., Linica, A., Della-Casa, M. S., Kamiguti, A. S., Ho, P. L., Crampton, J. M., and Theakston, R. D. (1999) Arch. Biochem. Biophys. 369, 295-301.

9. Hung, C.-C., Huang, K.-F., and Chiou, S.-H. (1994) Biochem. Biophys. Res. Commun. 205, 1707-1715.

10. Laemmli, U. K. (1970) Nature 227, 680- 685.

11. Rosenthal, J . A., Hsu, S. H., Schneider, D., Gentile, L. N., Messier, N. J ., Vaslet, C. A., and Hawrot, E. (1994) J . Biol. Chem. 269, 11178-11185.
12. Ouyang, C. (1957) J . Formosan Med. Assoc. 56, 435- 448.

13. Meaume, J . (1966) Toxicon 4, 25-58.

14. Bjarnason, J. B., Barish, A., Direnzo, G. S., Campbell, R., and Fox, J. W. (1983) J . Biol. Chem. 258, 12566-12573.

15. Ewart, M. R., Hatton, M. W. C., Bosford, J. M., and Dodgson, K. S. (1970) Biochem. J . 118, 603-609.

16. Holleman, W. H., and Weiss, L. J . (1976) J . Biol. Chem. 251, 1663-1669.

17. Dobeli, H., Trecziak, A., Gillessen, D., Matile, H., Srivastava, I. K., Perrin, L. H., J akob, P. E., and Certa, U. (1990) Mol. Biochem. Parasitol. 41, 259-268.

18. Pan, H., Du, X., Yang, G., Zhou, Y., and Wu, X. (1999) Biochem. Biophys. Res. Commun. 255, 412- 415. 